\title{
Les liaisons dangereuses: immunological synapse formation in animals and plants
}

\author{
Chian Kwon, Ralph Panstruga and Paul Schulze-Lefert
}

Max-Planck-Institut für Züchtungsforschung, Department of Plant Microbe Interactions, D-50829 Köln, Germany

The immunological synapse in vertebrates describes a specialized junction between a $\mathrm{T}$ cell and a target cell, enabling execution of immune responses through focal secretion. Recent insights in the plant immune system suggest that plant cells assemble a pathogen-inducible machinery at the cell surface that shares several features with the immunological synapse. Apparent mechanistic commonalities include co-stimulatory non-self alarm signals as triggers, cell polarization driven by actin cytoskeleton remodeling, protein concentration into ring-shaped assemblies at the cell periphery and focal exocytosis mediated by soluble $\boldsymbol{N}$-ethylmaleimide sensitive factor attachment protein receptor (SNARE) proteins that are core factors for vesicle fusion. Although in plants, execution of immune responses by polar secretion seems to be a cell type-independent property, its confinement to $T$ cells in the vertebrate immune system might reflect a greater division of labor.

\section{Introduction}

In vertebrates, lymphocytes play critical roles in cellmediated immunity against pathogens. On the detection of non-self molecular structures on cell surfaces, a subset of these, including cytotoxic T cells (CTLs), natural killer (NK) cells and natural killer T cells (NKTs), release cytolytic and other cell killing molecules to destroy their target cells, but spare themselves from being killed [1]. The delivery of cytotoxic cargo occurs at contact sites of CTLs and NK cells with their respective target cells, where a specialized and localized structure, the immunological synapse, is formed (see below). Immunological synapses are also established between $\mathrm{T}$ helper cells that express the CD4 glycoprotein (CD4 ${ }^{+}$cells) and professional or nonprofessional antigen presenting cells. However, cargo secreted by the $\mathrm{T}$ helper cells is not cytotoxic but is made up of cytokines that stimulate further immune cells such as macrophages and cytotoxic T cells. Although the molecular composition of both types of immunological synapses is distinct, they share biochemical and functional similarities [2]. Plants lack specialized immune cell patrols and rely on the capacity of each cell to recognize and mount effective immune responses against potential invaders. Plants evolved two radar systems for non-self recognition: one comprises pattern recognition receptors (PRRs) on the

Corresponding author: Schulze-Lefert, P. (schlef@mpiz-koeln.mpg.de). cell surface, which detect conserved microbe-associated molecular patterns (MAMPs). A second class of largely intracellular sensors, called resistance $(\mathrm{R})$ proteins, recognize polymorphic, typically isolate-specific pathogen effectors. Unlike PRR-triggered immune responses, immunity conferred by $\mathrm{R}$ proteins is frequently associated with a rapid suicide of the host cells. Although structural similarities between plant pattern receptors and animal innate immunity receptors have been described before [3-6], potential mechanistic parallels in the execution of immune responses in the two kingdoms have not been explored.

Cell autonomous immune responses are widespread in plant-microbe interactions and often terminate a pathogen attack at the cell surface, without affecting host cell viability. Here we discuss recent findings on the formation of a pathogen-induced secretion machinery in plant cells that stops parasite growth at the cell surface. We contrast these events with processes underlying $\mathrm{T}$ cell-mediated immunological synapse formation in vertebrates. Although the former involves the formation of a cellular junction between a plant and pathogen cell and the latter a junction between two host cells, we argue that these processes can be considered as a realization of cell-mediated immunity in the two kingdoms that share fundamental mechanistic features through localized recruitment of functionally similar molecules.

\section{Non-self recognition triggers immune responses in plants and animals}

The critical contact leading to $\mathrm{CD} 4^{+} \mathrm{T}$-cell activation is provided by binding of the plasma membrane-resident $\mathrm{T}$ cell receptor (TCR) to a short antigen peptide presented by major histocompatibility complex proteins (MHC) on the surface of another cell [e.g. a professional antigen presenting cell (APC)] [7]. This interaction is enhanced by the plasma membrane-resident CD4 co-receptor that binds to other portions of peptide-presenting MHC molecules $[7,8]$. Activation of naïve $\mathrm{T}$ cells needs a co-stimulatory second signal provided by the CD28 membrane receptor, which binds CD80 (B7.1) and CD86 (B7.2) proteins on the APC surface $[9,10]$. CD80 and CD86 are expressed at low levels on resting APCs and are upregulated after activation $[9,10]$. The signal triggered by the TCR-peptide-MHC interaction is transduced by a tightly controlled phosphorylation cascade through tyrosine phosphorylation of 
the immunoreceptor tyrosine-based activation motifs (ITAMs) in the TCR complex [11]. Additionally, integrins such as leucocyte function associated antigen (LFA-1) seem to stabilize the immunological synapse [12]. Ultimately, this leads to the activation of transcription factors in-

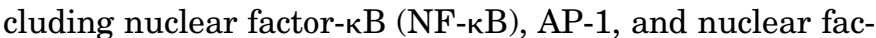
tor of activated $\mathrm{T}$ cells (NFAT) to induce activation, differentiation, proliferation and effector function of $\mathrm{T}$ cells $[13,14]$.

Two plant PRRs, Arabidopsis flagellin sensing 2 (FLS2) and Elongation factor Tu receptor (EFR), have been identified, each composed of structural modules permitting perception of extracellular MAMPs and subsequent initiation of intracellular signaling. Both receptors contain extracellular leucine-rich repeats (LRRs), a single transmembrane-spanning helix, and an intracellular kinase domain. FLS2 directly binds to the flg22 peptide, the most conserved part of the bacterial motor protein flagellin, whereas EFR recognizes elf18, an acetylated 18-mer peptide derived from the $\mathrm{N}$ terminus of bacterial elongation factor EF-Tu [15-17]. Because both receptors contain an intracellular kinase module, signal transduction is likely initiated through phosphorylation cascades [18] after binding of the cognate MAMPs to the extracellular LRR region. Recent findings have shown that flg22 signal transduction through FLS2 involves in vivo complex formation of FLS2 with BRI1-associated receptor kinase 1 (BAK1), an LRRcontaining receptor-like kinase that was originally identified as an adaptor or co-receptor of the brassinosteroid hormone receptor BRI1 [19]. This suggests that BAK1 might function as an adaptor or co-receptor for the function of various surface receptors. In rice, a chitin elicitor binding protein ( $\mathrm{CEBiP})$ binds one of the major constituents of fungal cell walls, $N$-acetyl-chitooligosaccharide oligomers. The plasma membrane-anchored CEBiP contains two extracellular LysM domains, a module implicated in peptidoglycan-binding, but lacks an intracellular kinase domain, whereas the recently identified Arabidopsis chitin elicitor receptor kinase 1 (CERK1) contains both three extracellular LysM domains and a cytoplasmic kinase motif [20-22]. One possibility is that rice CEBiP functions as co-receptor by binding to a yet unidentified receptor-like kinase.

Application of the above described MAMPs changes the expression profile of $\sim 3 \%$ of tested Arabidopsis genes within $30 \mathrm{~min}$. These encode a disproportionate number of transcription factors, protein kinases and phosphatases, receptor-like kinases, and proteins regulating protein turnover. Because these changes are also seen in the presence of cycloheximide (an inhibitor of protein biosynthesis), MAMP-triggered transcriptional reprogramming likely involves rapidly turned-over repressors [23]. Of note, elf18, flg22 or chitin treatment each change the expression of a largely overlapping set of genes, suggesting convergence of signaling pathways triggered by different MAMP receptors [17,23,24]. Thus, analogous to T cells, extracellular non-self signals are in plants rapidly processed to reprogram the transcriptional machinery for immune responses. A highly diversified family of WRKY DNA binding domain-containing transcription factors has been implicated in MAMP-triggered transcriptional reprogramming events [18,25-29]. Recent findings suggest that a disease resistance response that is triggered by an intracellular $\mathrm{R}$ sensor involves potentiation of PRR-triggered immune responses. This requires nuclear activity of the $\mathrm{R}$ sensor and its ligand-dependent direct association and interference with WRKY repressors that exert negative feedback control on PRR-triggered defense responses [29].

\section{Establishment of the immunological synapse}

Although $\mathrm{CD}^{+} \mathrm{T}$ cells are capable of detecting a single agonist-peptide MHC complex on a target cell, approximately ten complexes are needed to stop T-cell migration and to initiate the formation of a stable APC contact site [30]. Ligated TCR complexes become, within minutes after an initial APC encounter, relocalized and accumulate together with the co-stimulatory CD28 receptor at the nascent synapse [31]. This protein relocalization in the plasma membrane is part of a complex molecular segregation process leading to the formation of spatially distinct supramolecular activation clusters (SMACs) with a 'bulls-eye' zone pattern at contact sites between $\mathrm{T}$ cells and target cells (Figure 1a-d) [32-35]. The segregation process of distinct SMACs (called central and peripheral SMACs) requires both TCR ligation and co-stimulation [36,37], is energy-dependent and involves mass transport of randomly distributed lipid raft-like microdomains on the T-cell surface to APC contact sites through cytoskeletal linkage and molecular motors (Figure 1e) $[35,37,38]$. Formation of SMACs and maturation of the nascent synapse seems to be driven by cytoskeletal polarization, which is accompanied by the movement of the microtubule-organizing center (MTOC) and Golgi apparatus (GA) beneath contact sites, thereby enabling directed release of cytotoxic granules and cytokines as well as localized TCR internalization [39-41]. Numerous TCR proximal signaling molecules are needed for actin accumulation and F-actin dynamics at the immunological synapse $[34,41]$. The guanine-nucleotide-exchange factor (GEF) VAV1 plays a key role in this process and is thought to catalyze the exchange of GDP for GTP on the RHO GTPases CDC42 and RAC1, as well as recruiting the large GTPase dynamin-2 to the immunological synapse $[42,43]$. Wiskott-Aldrich syndrome protein (WASP) and WASP-family verprolin homologous protein (WAVE) are key proteins needed for actin cytoskeleton re-organization by activating Arp2/3 complex activity, which in turn nucleates actin filaments [44-46]. CDC42 activates WASP by direct interaction with the auto-inhibitory domain of WASP, and RAC1 activates WAVE1 by association with an inhibitory protein complex, thereby releasing active WAVE1 [47-50]. Despite these advances, the exact roles and action sites of actin-regulatory and actin-nucleating proteins during T-cell activation and their spatial distribution during maturation of the immunological synapse are still ill-defined.

Although TCR-peptide-MHC complexes are highly concentrated in the center of the immunological synapse, initial signaling to induce the formation of the immunological synapse is transient [33,35]. Rather than amplifying the signal, the synapse controls the signal strength by recruiting and degrading signaling molecules to fully 


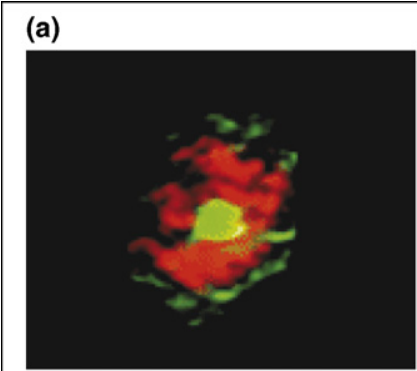

(c)

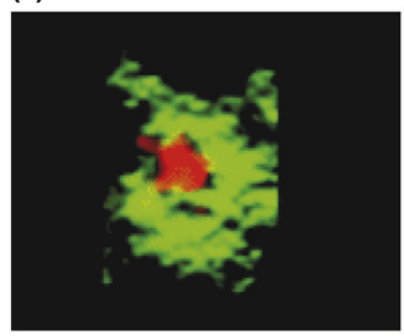

(b)

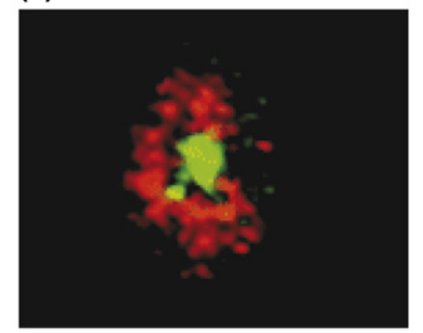

(d)

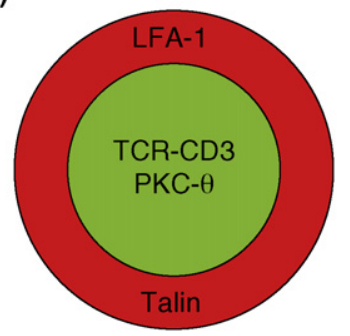

(e)

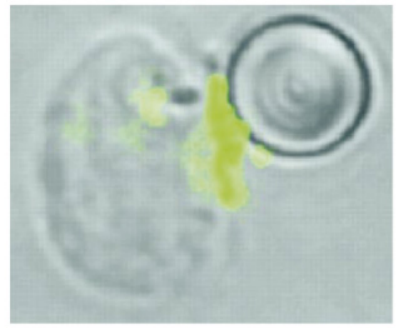

$\overline{T R E N D S ~ i n ~ I m m u n o l o g y ~}$

Figure 1. Spatial rearrangement of proteins at contact sites between $T$ cells and target cells. At the immunological synapse of a $\mathrm{CD}^{+} \mathrm{T}$ cell with an antigen presenting cell, T cell receptor (TCR)-CD3 complex [green in (a) and (b)] is centrally concentrated and differentially localized from talin [red in (a)] and leucocyte function associated antigen (LFA-1) [red in (b)]. Protein kinase $C \theta$ (PKC- $\theta)$ [red in (c)] also accumulates in the center of the synapse [talin, green in (c)] [32]. (d) Molecular segregation results in a 'bulls-eye' pattern at the immunological synapse. TCR-CD3 complex and PKC- $\theta$ are localized in the central supramolecular activation cluster (cSMAC, green), and talin and LFA-1 accumulate in the peripheral SMAC (pSMAC, red). (e) Co-stimulation-dependent clustering of sterol-rich lipids raft (green) at the contact site between a CD4 ${ }^{+} \mathrm{T}$ cell and a polystyrene microbead coated with anti-CD3 and anti-CD28 antibodies. The lipid raft was visualized by staining GM1 glycosphingolipid with fluorescein isothiocyanate-labeled cholera toxin subunit b [37]. Adapted, with permission, from Refs. [32,37].

activate $\mathrm{T}$ cells [51-53]. Toward this end, the polarized cytoskeleton plays a key role in the recruitment of signaling components to the synapse because inhibition of actin polymerization in preformed $\mathrm{CD} 4^{+} \mathrm{T}$ cell-APC contacts abrogates T-cell activation [51-53].

\section{Pathogen-induced cell polarization and focal secretion in plants}

Plant cells respond to surface colonization by phytopathogenic bacteria or attempted entry of filamentous parasites such as fungi or oomycetes (filamentous protists) with rapid cell polarization and secretion, including polar alignment of the actin cytoskeleton and directed movement of organelles to pathogen contact sites [54-57]. Visualization of plant actin cytoskeleton dynamics by green fluorescent protein (GFP) fused to the actin-binding domain of human talin in interactions between Arabidopsis and oomycete parasites showed extensive host cytoskeleton remodeling,

leading to the formation of large polarized actin bundles beneath pathogen entry sites [56]. Drug-mediated or genetic perturbation of actin dynamics permitted effective parasite entry [58,59]. Expression of a constitutively active Rac/Rop GTPase modulates actin cytoskeleton rearrangements beneath pathogen contact sites and increased fungal entry [60,61]. Although plants contain homologs of ARP2/ 3 , WASP and WAVE complex components, their potential contribution to pathogen-induced cell polarization remains to be tested.

Pathogen-triggered cell polarization typically leads to the formation of a ring-shaped and stable subcellular structure of $\sim 50-\mu \mathrm{m}$ diameter, designated papilla (Figure 2a) [62]. Papillae are unique to plant cells because they are generated by focal deposition of de novo synthesized cell wall material, including callose, a $(1 \rightarrow 3)-\beta$-Dglucan, between the inner cell wall and the plasma membrane (paramural space) [63]. The multilayered appearance of papillae is indicative of a sequential order of cell wall deposits generated by the focal release of cell wall precursors. One hypothesis is that pathogen-inducible cell wall appositions provide a structural scaffold for antimicrobial compounds and contribute to structural reinforcement of the cell wall against parasite ingress. However, specific depletion of papillary callose in pmr4/ gsl5 Arabidopsis mutants, carrying null mutations in 1 of 12 callose synthase-like family members, results in constitutive activation of the salicylic acid (SA)-dependent defense pathway and enhanced disease resistance responses [64,65]. This suggests a more subtle role of papillary cell wall components such as negative regulation of the SA defense pathway by plasma membrane-resident PMR4/GSL5 callose synthase or by its enzymatic product.

The isolation of Arabidopsis mutants that are partially immunocompromised against ascomycete powdery mildew fungi revealed novel components required for focal immune responses at the cell periphery. The penetration 1 (PEN1) syntaxin, peroxisomal PEN2 $\beta$-glycosyl hydrolase and the PEN3 ABC transporter are each necessary to restrict powdery mildew entry coincident with the formation of papillae [66-68]. PEN2 and PEN3 act in the same pathway and are implicated in the cytoplasmic synthesis and transport of small antimicrobial compounds across the plasma membrane, respectively [67,68]. The plasma membrane-resident PEN1 syntaxin acts in a second pathway $[66,67]$ and has recently been shown to mediate vesicle fusion processes by forming secretory ternary SNARE complexes together with the adaptor protein synaptosomal-associated protein of $33 \mathrm{kDa}$ (SNAP33) and vesicle-associated membrane protein (VAMP) 721 or VAMP722 [69]. Each of these proteins is subject to dynamic relocalization on parasite attack and becomes concentrated beneath incipient pathogen entry sites. This includes concentration of PEN1 syntaxin and associated SNAP33, as well as of the PEN3 ABC transporter in a seemingly sterol-enriched lipid raft-like plasma membrane micro-domain (Figure 2b, c and e) [68-71]. Residence of the PEN1 syntaxin in lipid raft-like microdomains is further supported by a partial, sterol-dependent association of the SNARE protein with detergent-resistant membranes (N. Zappel and R.P., unpublished), which are widely 


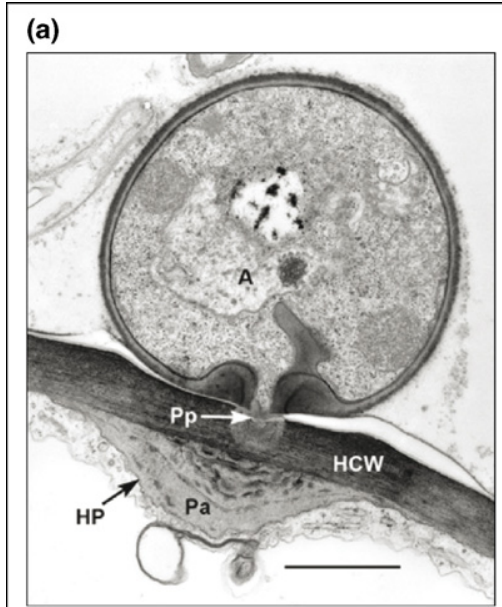

(c)

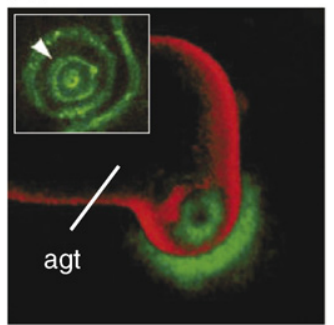

(e)

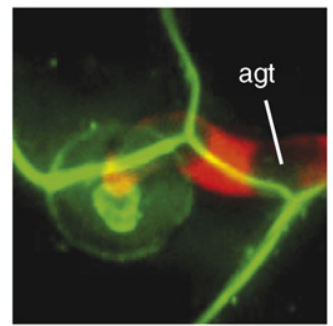

(b)

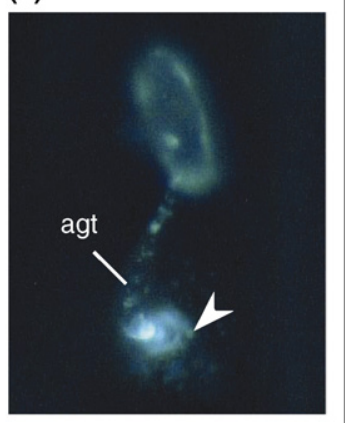

(d)

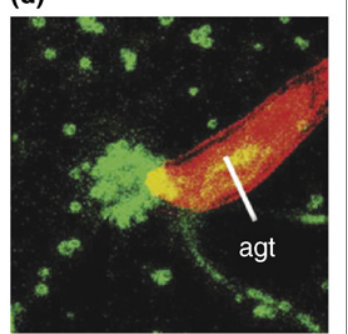

(f)

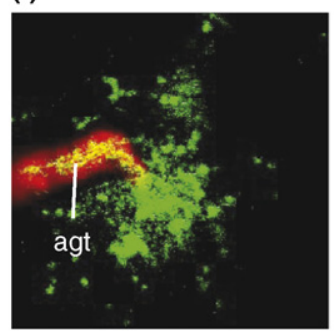

$\overline{\text { TRENDS in Immunology }}$

Figure 2. Focal concentration of secretion-related gene products of plants at incipient pathogen entry sites. (a) Transmission electron micrograph showing penetration of a cucumber epidermal cell by an appressorium (A) of the fungus Colletotrichum lagenarium. The fungal penetration peg $(\mathrm{Pp})$ emerging from the base of the appressorium has breached the plant cuticle but has become restricted within the host cell wall ( $\mathrm{HCW}$ ), and a callose papilla $(\mathrm{Pa})$ has been deposited by the host cell at the site of attempted penetration. HP, host plasma membrane. Bar $=1 \mu \mathrm{m}$ [62]. (b) Filipin-mediated fluorescence at the tip of the fungus Blumeria graminis appressorial germ tube (agt) on the leaf surface and underneath in the attacked host epidermal cell (arrowhead). Filipin staining indicates stero accumulation in membranes and might represent clusters of lipid rafts (indicated by the arrowhead) [71]. Focal accumulation of penetration 1 (PEN1) (c), PEN2 (d), PEN3 (e) and VAMP722 (f) (green) at the contact site between plant and fungal cells (red) $[67,70]$. Transgenic plants expressing functional green fluorescent protein (GFP)-fused proteins were inoculated with $B$. graminis conidiospores, and the interaction sites were analyzed by confocal microscopy. A typical 'bulls eye' pattern of GFP-PEN1 accumulation beneath a fungal attack site is shown in the inset of (c) The arrowhead points to a presumed endomembrane compartment [70]. agt, appressorial germ tube. Adapted, with permission, from Refs. $[62,67,70,71]$.

considered as a biochemical approximation of lipid rafts [72]. In addition, PEN2-associated peroxisomes and VAMP722-containing vesicles directionally move to and congregate under pathogen portals (Figure $2 \mathrm{~d}$ and f) [67]. However, unlike in animals where peroxisomes shuttle on microtubules, plant peroxisomes move exclusively along actin filaments, and pharmacological interference with the actin cytoskeleton leads to a rapid arrest of peroxisome movement [73-75].

Functional GFP-PEN1 and PEN3-GFP each accumulate in concentric 'bulls-eye' rings in the region of the host plasma membrane under a fungal appressorium (the tip of the fungal germ tube that adheres tightly to the plant cell wall and produces a needle-like peg to puncture the host cell wall; Figure 2a), suggesting a supramolecular organization that is reminiscent of T cell SMACs (Figure 2c and e) [32,68,70]. GFP-PEN1 was additionally located in the apparent interior of cell wall containing papillae [70]. Because electron micrographs have shown the presence of membranebound vesicles within papilla [76-78], this hints to unusual trafficking events and extrusion of vesicles in the paramural space, some of which might become trapped in the papillary cell wall scaffold. The directed movement of GFP-VAMP722 vesicles to pathogen portals and a pen phenocopy seen in VAMP $721^{+/-}$VAMP $722^{-1-}$ mutant plants is consistent with the hypothesis that plasma membrane-resident PEN1 syntaxin acts through the formation of heterooligomeric SNARE complexes, enabling focal secretion of vesicle cargo at fungal entry sites [69]. Likewise, a distinct syntaxin family member, SYP132, has been shown to function in secretion-based antibacterial defense [79], suggesting that secretory processes are a general feature of plant immune responses against different pathogens classes but act through distinct pathways. A bacterial virulence factor, HopM1, was shown to interfere with the plant secretory system by degrading an adenosine diphosphate ribosylation factor guanine nucleotide exchange factor (ARF-GEF) [80] .

\section{Killing cargo}

A delay in the timing of papilla formation both in pen1 mutants [70] and in transgenic lines in which VAMP721 and VAMP722 are constitutively co-silenced suggests cell wall precursors and/or enzymes involved in papillary cell wall biosynthesis as potential vesicle cargo [69]. This and the genetic analysis of pen double mutants points to the combined action of SNARE-mediated discharge of VAMP721 and VAMP722 vesicle cargo and PEN3 ABC transporter-driven translocation of small antimicrobial compounds for a maximal immune response [67-69]. Like CTL-, NK- and NKT-dependent immune responses, plant cells executing PEN-dependent immune responses spare themselves from being killed. Possibly, formation of the papillary cell wall scaffold together with focal secretion enables containment of antimicrobials and contributes to a self-protection mechanism. However, a precise understanding of the toxic principle(s) underlying PEN-dependent termination of pathogenesis at the cell periphery await comprehensive compiling of VAMP721 and VAMP722 vesicle cargo and structure determination of molecules translocated by PEN3.

In animals, the lytic activity of CTLs and NK cells is known to be compartmentalized in specialized granules in their cytoplasm. Focal secretion of 'secretory lysosomes' at contact sites formed between these immune cells and their targets selectively induces target cell death $[1,81,82]$. This activity requires perforin, a soluble protein that is related to the pore-forming C9 component of complement, suggesting that secreted perforin acts through pore formation and disruption of the target plasma membrane [8385]. Other components of lytic granules, such as the serine 
proteases granzyme A and granzyme B, require perforin for their activity, possibly by facilitating the entry to target cells through a perforin pore either on the plasma membrane and/or endosomes to trigger apoptosis in target cells through caspase cleavage [83-85]. Increasingly more is becoming known about the molecular machinery that regulates the docking and fusion of this organelle with the plasma membrane. Recent insight comes from studies on inherited disorders in humans leading to defects in the granule-dependent cytotoxic function of lymphocytes $[1,81,82]$. Whereas mutations in $R A B 27 A$ causing loss of function of the small GTPase Rab27a lead to defective granule docking $[86,87]$, mutations in $U N C 13 D$ causing loss of hMunc13-4 function impair the fusion of cytotoxic granules with target membrane that have docked at the immunological synapse [88]. A third component that has been genetically linked to granule-dependent dysfunction of NK cells is loss-of-function mutations in STX11 encoding a syntaxin family member [89]. The involvement of Rab27a, hMunc13-4 and syntaxin 11 in vesicle fusion strongly suggests the engagement of SNAREs in cytotoxic granule exocytosis in cytolytic T cells. Interestingly, cytotoxic executor proteins such as perforin and granzyme B and fusion regulatory proteins Rab27a and hMunc13-4 localize on distinct vesicular structures [90]. Apparently only at the last step of the exocytic pathway do both types of vesicles fuse near the plasma membrane [90]. In this context, it is tempting to speculate that the observed size variation of Arabidopsis GFP-VAMP722-tagged vesicles close to the plasma membrane (Figure 2f) reflects a similar mechanism of compound exocytosis in which vesicles fuse with each other before their fusion with the plasma membrane [91]. In addition, SNAREs seem to be engaged in TCR recycling and the secretion of cytokines in activated $\mathrm{CD}^{+} \mathrm{T}$ cells, and it is likely that these are different family members from those involved in the secretion of cytotoxic granules [53,82].

\section{Co-stimulation is required for focal defense responses}

Integrated functional and cell biological studies of plant PRRs have just begun. Thus, it is not yet known whether these receptors cluster at sites of attempted fungal ingress or beneath bacterial colonies. However, a GFP-tagged functional fusion protein of the plasma membrane-resident Arabidopsis FLS2 PRR, which detects bacterial flagellin, becomes within minutes endocytosed and degraded in a ligand-dependent manner [92]. Because these experiments involved treatment of plant cells with a single stimulus, the flg22 peptide, it should be interesting to study FLS2 localization underneath surface colony-forming pathogenic Pseudomonas cells. Direct evidence that MAMP treatment alone is insufficient to induce the characteristic cell polarization process seen in bacterial interactions or those with filamentous parasites was demonstrated by co-stimulation

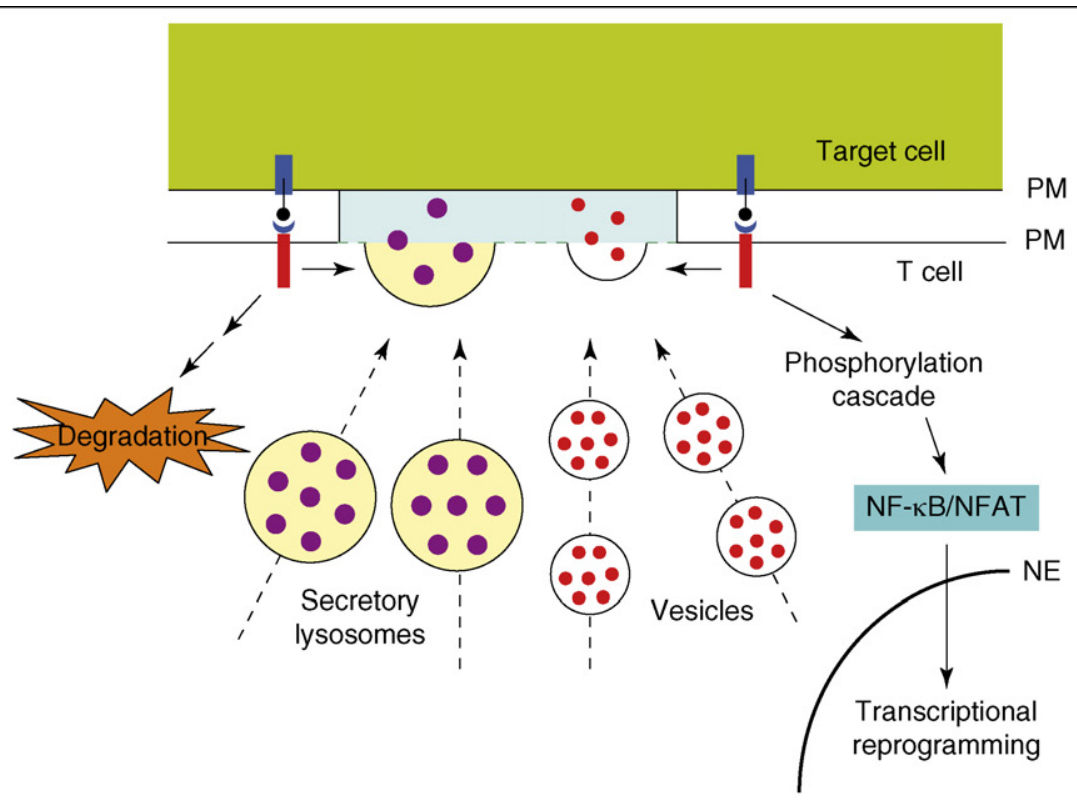

\begin{tabular}{|lll|}
\hline Key: & & \\
& Immunological synapse & - Toxic molecules (perforinand granzyme) \\
T cell receptor & $\bullet$ & Cytokines \\
MHC-Antigen peptide complex & i & Polarized cytoskeleton \\
- Lipid-raft-like membrane & I & \\
\hline
\end{tabular}

$\overline{\text { TRENDS in Immunology }}$

Figure 3. Schematic overview of the immunological synapse in cell-to-cell interactions in vertebrates and plants. A cartoon depicting key cellular processes for immunological synapse function in vertebrate $T$ cells. The immunological synapse forms a tight interface between a $T$ and a target cell and provides a scaffold for focal secretion of secretory lysosomes and vesicles releasing cytotoxic molecules (as in the case of cytotoxic T cells) or cytokines (as in the case of CD $4^{+}$cells), respectively. The strength of signaling at the immunological synapse is controlled by continuous delivery and degradation of receptors. 
experiments of individual plant cells with a MAMP from an oomycete pathogen (Pep-25) and mechanical stimulation using a needle of the same diameter as a fungal hypha [93]. Whereas mechanical stimulation alone induced polarized migration of intracellular organelles and the production of reactive oxygen intermediates (ROS), MAMP treatment induced ROS accumulation and the activation of a subset of defense-related genes [93]. However, co-stimulation triggered most of the early responses seen in interactions on fungal attack but was still insufficient to trigger papilla formation [93]. This finding supports the idea that execution of polarized resistance responses in individual plant cells demands integration of stimulatory signals in much the same way as co-stimulation is needed for the establishment of a stable immunological synapse. Because it is likely that a host cell detects an array of pathogen-derived MAMPs, a single MAMP such as Pep-25 may trigger only a subset of the processes seen in the interaction between a host and pathogen cell.

\section{Conclusion}

Pattern recognition receptors in plants and animals are composed of similar structural protein modules [3-6]. This is, however, unlikely to reflect a common evolutionary origin but is likely a consequence of biochemical constraints to build non-self sensors from a limited number of eukaryotic protein modules [94]. Likewise, the similarities discussed above in cell-mediated immunity in plants and animals are likely to reflect shared biochemical and cellular constraints that demand integration of non-self sensor perception in

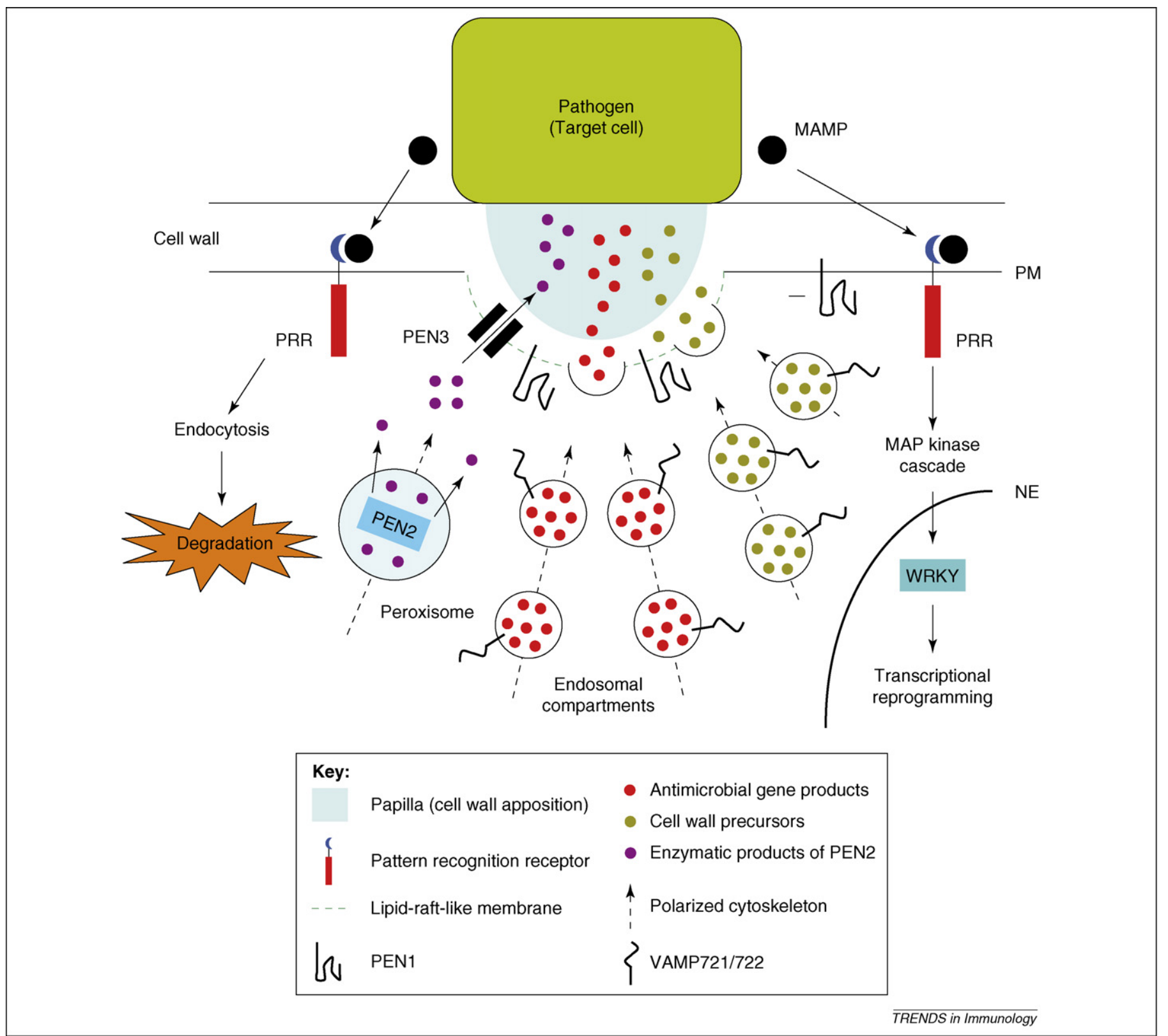

Figure 4. Schematic overview of the immunological synapse in cell-to-cell interactions in vertebrates and plants. Speculative scheme illustrating focal secretion at contact sites between plant and pathogen (target) cells. Although the clustering of plant pattern recognition receptors at pathogen contact sites remains to be tested, these sites might provide a platform for recognition of microbe-associated molecular patterns and for secretion of antimicrobials through vesicles and translocation of small toxic molecules produced by peroxisome-associated penetration 2 (PEN2). Genetic studies showed that the PEN1-dependent secretory pathway functions independently from the PEN2/PEN3 pathway $[67,68]$. PM, plasma membrane; NE, nuclear envelope. 
single cell systems for controlled focal delivery of immune executors (Figures 3,4). In this context, we believe that future research on cell-mediated immunity in animals and plants will be mutually beneficial.

\section{Acknowledgements}

We thank J. Howard for critical comments and helpful discussions and R. O'Connell, S. Somerville and H. S. Yun for providing Figure 2a, Figure $2 \mathrm{e}$ and Figure 2f, respectively. This work was supported by funds from the Deutsche Forschungsgemeinschaft SPP1212 (Plant-Microbe) to C.K. and P.S.-L. and SFB670 (Cell-Autonomous Immunity) to R.P. and P.S.-L.

\section{References}

1 Stinchcombe, J.C. and Griffiths, G.M. (2007) Secretory mechanisms in cell-mediated cytotoxicity. Annu. Rev. Cell Dev. Biol. 23, 495-517

2 Stinchcombe, J.C. et al. (2001) The immunological synapse of CTL contains a secretory domain and membrane bridges. Immunity 15 , $751-761$

3 Nurnberger, T. et al. (2004) Innate immunity in plants and animals: striking similarities and obvious differences. Immunol. Rev. 198, 249266

4 Espinosa, A. and Alfano, J.R. (2004) Disabling surveillance: bacterial type III secretion system effectors that suppress innate immunity. Cell. Microbiol. 6, 1027-1040

5 Zipfel, C. and Felix, G. (2005) Plants and animals: a different taste for microbes? Curr. Opin. Plant Biol. 8, 353-360

6 Dardick, C. and Ronald, P. (2006) Plant and animal pathogen recognition receptors signal through non-RD kinases. PLoS Pathog. $2, \mathrm{e} 2$

7 Rudolph, M.G. et al. (2006) How TCRs bind MHCs, peptides, and coreceptors. Annu. Rev. Immunol. 24, 419-466

8 Konig, R. (2002) Interactions between MHC molecules and co-receptors of the TCR. Curr. Opin. Immunol. 14, 75-83

9 Sansom, D.M. et al. (2003) What's the difference between CD80 and CD86? Trends Immunol. 24, 314-319

10 Acuto, O. and Michel, F. (2003) CD28-mediated co-stimulation: a quantitative support for TCR signaling. Nat. Rev. Immunol. 3, 939-951

11 Pitcher, L.A. and van Oers, N.S. (2003) T-cell receptor signal transmission: who gives an ITAM. Trends Immunol. 24, 554-560

12 Sims, T.N. and Dustin, M.L. (2002) The immunological synapse: integrins take the stage. Immunol. Rev. 186, 100-117

13 Bonizzi, G. and Karin, M. (2004) The two NF-кB activation pathways and their role in innate and adaptive immunity. Trends Immunol. 25, 280-288

14 Macian, F. (2005) NFAT proteins: key regulators of T-cell development and function. Nat. Rev. Immunol. 5, 472-484

15 Gomez-Gomez, L. and Boller, T. (2000) FLS2: an LRR receptor-like kinase involved in the perception of the bacterial elicitor flagellin in Arabidopsis. Mol. Cell 5, 1003-1011

16 Zipfel, C. et al. (2004) Bacterial disease resistance in Arabidopsis through flagellin perception. Nature 428, 764-767

17 Zipfel, C. et al. (2006) Perception of the bacterial PAMP EF-Tu by the receptor EFR restricts Agrobacterium-mediated transformation. Cell $125,749-760$

18 Asai, T. et al. (2002) MAP kinase signalling cascade in Arabidopsis innate immunity. Nature 415, 977-983

19 Chinchilla, D. et al. (2007) A flagellin-induced complex of the receptor FLS2 and BAK1 initiates plant defense. Nature 448, 497-500

20 Bateman, A. and Bycroft, M. (2000) The structure of a LysM domain from E. coli membrane-bound lytic murein transglycosylase D (MltD). J. Mol. Biol. 299, 1113-1119

21 Kaku, H. et al. (2006) Plant cells recognize chitin fragments for defense signaling through a plasma membrane receptor. Proc. Natl. Acad. Sci. U. S. A. 103, 11086-11091

22 Miya, A. et al. (2007) CERK1, a LysM receptor kinase, is essential for chitin elicitor signaling in Arabidopsis. Proc. Natl. Acad. Sci. U. S. A. 104, 19613-19618

23 Navarro, L. et al. (2004) The transcriptional innate immune response to flg22. Interplay and overlap with Avr gene-dependent defense responses and bacterial pathogenesis. Plant Physiol. 135, 1113-1128

24 Ramonell, K. et al. (2005) Loss-of-function mutations in chitin responsive genes show increased susceptibility to the powdery mildew pathogen Erysiphe cichoracearum. Plant Physiol. 138, 10271036

25 Ulker, B. and Somssich, I.E. (2004) WRKY transcription factors: from DNA binding towards biological function. Curr. Opin. Plant Biol. 7 , 491-498

26 Chisholm, S.T. et al. (2006) Host-microbe interactions: shaping the evolution of the plant immune response. Cell 124, 803-814

$27 \mathrm{Xu}$, X. et al. (2006) Physical and functional interactions between pathogen-induced Arabidopsis WRKY18, WRKY40, and WRKY60 transcription factors. Plant Cell 18, 1310-1326

28 Journot-Catalino, N. et al. (2006) The transcription factors WRKY11 and WRKY17 act as negative regulators of basal resistance in Arabidopsis thaliana. Plant Cell 18, 3289-3302

29 Shen, Q.H. et al. (2007) Nuclear activity of MLA immune receptors links isolate-specific and basal disease-resistance responses. Science 315, 1098-1103

30 Irvine, D.J. et al. (2002) Direct observation of ligand recognition by $\mathrm{T}$ cells. Nature 419, 845-849

31 Pentcheva-Hoang, T. et al. (2004) B7-1 and B7-2 selectively recruit CTLA-4 and CD28 to the immunological synapse. Immunity 21, 401413

32 Monks, C.R.F. et al. (1998) Three-dimensional segregation of supramolecular activation clusters in T cells. Nature 395, 82-86

33 Huppa, J.B. and Davis, M.M. (2003) T-cell-antigen recognition and the immunological synapse. Nat. Rev. Immunol. 3, 973-983

34 Cemerski, S. and Shaw, A. (2006) Immune synapses in T-cell activation. Curr. Opin. Immunol. 18, 298-304

35 Saito, T. and Yokosuka, T. (2006) Immunological synapse and microclusters: the site for recognition and activation of $\mathrm{T}$ cells. Curr. Opin. Immunol. 18, 305-313

36 Wulfing, C. and Davis, M.M. (1998) A receptor/cytoskeletal movement triggered by costimulation during T cell activation. Science 282, 22662269

37 Viola, A. et al. (1999) T lymphocyte costimulation mediated by reorganization of membrane microdomains. Science 283, 680-682

38 Bromley, S.K. et al. (2001) The immunological synapse. Annu. Rev. Immunol. 19, 375-396

39 Geiger, B. et al. (1982) Spatial relationships of microtubule-organizing centers and the contact area of cytotoxic T lymphocytes and target cells. J. Cell Biol. 95, 137-143

40 Kupfer, A. and Dennert, G. (1984) Reorientation of the microtubuleorganizing center and the Golgi apparatus in cloned cytotoxic lymphocytes triggered by binding to lysable target cells. $J$. Immunol. 133, 2762-2766

41 Billadeau, D.D. et al. (2007) Regulation of T-cell activation by the cytoskeleton. Nat. Rev. Immunol. 7, 131-143

42 Tybulewicz, V.L.J. (2005) Vav-family proteins in T-cell signalling. Curr. Opin. Immunol. 17, 267-274

43 Gomez, T.S. et al. (2005) Dynamin 2 regulates T cell activation by controlling actin polymerization at the immunological synapse. Nat. Immunol. 6, 261-270

44 Takenawa, T. and Miki, H. (2001) WASP and WAVE family proteins: key molecules for rapid rearrangement of cortical actin filaments and cell movement. J. Cell Sci. 114, 1801-1809

45 Higgs, H.N. and Pollard, T.D. (2001) Regulation of actin filament network formation through Arp2/3 complex: activation by a diverse array of proteins. Annu. Rev. Biochem. 70, 649-676

46 Notarangelo, L.D. and Ochs, H.D. (2003) Wiskott-Aldrich Syndrome: a model for defective actin reorganization, cell trafficking and synapse formation. Curr. Opin. Immunol. 15, 585-591

47 Symons, M. et al. (1996) Wiskott-Aldrich Syndrome protein, a novel effector for the GTPase CDC42Hs, is implicated in actin polymerization. Cell 84, 723-734

48 Rohatgi, R. et al. (1999) The interaction between N-WASP and the Arp2/3 complex links Cdc42-dependent signals to actin assembly. Cell 97, 221-231

49 Eden, S. et al. (2002) Mechanism of regulating of WAVE1-induced actin nucleation by Rac1 and Nck. Nature 418, 790-793

50 Cory, G.O.C. and Ridley, A.J. (2002) Braking WAVEs. Nature 418, 732 733

51 Valitutti, S. et al. (1995) Sustained signaling leading to T cell activation results from prolonged $\mathrm{T}$ cell receptor occupancy: role of $\mathrm{T}$ cell actin cytoskeleton. J. Exp. Med. 181, 577-584 
52 Lee, K.H. et al. (2003) The immunological synapse balances T cell receptor signaling and degradation. Science 302, 1218-1222

53 Das, V. et al. (2004) Activation-induced polarized recycling targets T cell antigen receptors to the immunological synapse: involvement of SNARE complexes. Immunity 20, 577-588

54 Bushnell, W.R. and Zeyen, R.J. (1976) Light and electron microscope studies of cytoplasmic aggregates formed in barley cells in response to Erysiphe graminis. Can. J. Bot. 54, 1647-1655

55 Bestwick, C.S. et al. (1997) Localization of hydrogen peroxide accumulation during the hypersensitive reaction of lettuce cells to Pseudomonas syringae pv phaseolicola. Plant Cell 9, 209-221

56 Takemoto, D. et al. (2003) GFP-tagging of cell components reveals the dynamics of subcellular re-organization in response to infection of Arabidopsis by oomycete pathogens. Plant J. 33, 775-792

57 Koh, S. et al. (2005) Arabidopsis thaliana subcellular responses to compatible Erysiphe cichoracearum infections. Plant J. 44, 516-529

58 Kobayashi, Y. et al. (1997) Dynamic reorganization of microfilaments and microtubules is necessary for the expression of non-host resistance in barley coleoptile cells. Plant J. 11, 525-537

59 Miklis, M. et al. (2007) Barley MLO modulates actin-dependent and actin-independent antifungal defense pathways at the cell periphery. Plant Physiol. 144, 1132-1143

60 Schultheiss, H. et al. (2003) Functional analysis of barley RAC/ROP Gprotein family members in susceptibility to the powdery mildew fungus. Plant J. 36, 589-601

61 Opalski, K.S. et al. (2005) The receptor-like MLO protein and the RAC/ ROP family G-protein RACB modulate actin reorganization in barley attacked by the biotrophic powdery mildew fungus Blumeria graminis f.sp. hordei. Plant J. 41, 291-303

62 Tanaka, S. et al. (2007) Saccharomyces cerevisiae SSD1 orthologues are essential for host infection by the ascomycete plant pathogens Colletotrichum lagenarium and Magnaporthe grisea. Mol. Microbiol. 64, 1332-1349

63 Aist, J.R. (1976) Papillae and related wound plugs of plant cells. Annu. Rev. Phytopathol. 14, 145-163

64 Nishimura, M.T. et al. (2003) Loss of a callose synthase results in salicylic acid-dependent disease resistance. Science 301, 969-972

65 Jacobs, A.K. et al. (2003) An Arabidopsis callose synthase, GSL5, is required for wound and papillary callose formation. Plant Cell 15, 2503-2513

66 Collins, N.C. et al. (2003) SNARE-protein-mediated disease resistance at the plant cell wall. Nature 425, 973-977

67 Lipka, V. et al. (2005) Pre- and postinvasion defenses both contribute to nonhost resistance in Arabidopsis. Science 310, 1180-1183

68 Stein, M. et al. (2006) Arabidopsis PEN3/PDR8, an ATP binding cassette transporter, contributes to nonhost resistance to inappropriate pathogens that enter by direct penetration. Plant Cell $18,731-746$

69 Kwon, C. et al. (2008) Co-option of a default secretory pathway for plant immune responses. Nature 451, 835-840

70 Assaad, F.F. et al. (2004) The PEN1 syntaxin defines a novel cellular compartment upon fungal attack and is required for the timely assembly of papillae. Mol. Biol. Cell 15, 5118-5129

71 Bhat, R.A. et al. (2005) Recruitment and interaction dynamics of plant penetration resistance components in a plasma membrane microdomain. Proc. Natl. Acad. Sci. U. S. A. 102, 3135-3140

72 Brown, D.A. and London, E. (1998) Functions of lipid rafts in biological membranes. Annu. Rev. Cell Dev. Biol. 14, 111-136

73 Mano, S. et al. (2002) Distribution and characterization of peroxisomes in Araibdopsis by visualization with GFP: dynamic morphology and actin-dependent movement. Plant Cell Physiol. 43, 331-341

74 Jedd, G. and Chua, N.H. (2002) Visualization of peroxisomes in living plant cells reveals acto-myosin-dependent cytoplasmic streaming and peroxisome budding. Plant Cell Physiol. 43, 384-392

75 Mathur, J. et al. (2002) Simultaneous visualization of peroxisomes and cytoskeletal elements reveals actin and not microtubule-based peroxisome motility in plants. Plant Physiol. 128, 1031-1045

76 Zeyen, R.J. and Bushnell, W.R. (1979) Papilla response of barley epidermal cells caused by Erysiphe graminis: rate and method of deposition determined by microcinematography and transmission electron microscopy. Can. J. Bot. 57, 898-913

77 An, Q. et al. (2006) Multivesicular bodies participate in a cell wallassociated defense response in barley leaves attacked by the pathogenic powdery mildew fungus. Cell. Microbiol. 8, 1009-1019

78 An, Q. et al. (2006) Multivesicular compartments proliferate in susceptible and resistant $M L A 12$-barley leaves in response to infection by the biotrophic powdery mildew fungus. New Phytol. 172, $563-576$

79 Kalde, M. et al. (2007) The syntaxin SYP132 contributes to plant resistance against bacteria and secretion of pathogenesis-related protein 1. Proc. Natl. Acad. Sci. U. S. A. 104, 11850-11855

80 Nomura, K. et al. (2006) A bacterial virulence protein suppresses host innate immunity to cause plant disease. Science 313, 220-223

81 Bossi, G. and Griffiths, G.M. (2005) CTL secretory lysosomes: biogenesis and secretion of a harmful organelle. Semin. Immunol. $17,87-94$

82 Stow, J.L. et al. (2006) SNAREing immunity: the role of SNAREs in the immune system. Nat. Rev. Immunol. 6, 919-929

83 Voskoboinik, I. et al. (2006) Perforin-mediated target-cell death and immune homeostasis. Nat. Rev. Immunol. 6, 940-952

84 Pipkin, M.E. and Lieverman, J. (2007) Delivering the kiss of death: progress on understanding how perforin works. Curr. Opin. Immunol. 19, 301-308

85 Bolitho, P. et al. (2007) Apoptosis induced by the lymphocyte effector molecule perforin. Curr. Opin. Immunol. 19, 339-347

86 Menasche, G. et al. (2000) Mutations in RAB27A cause Griscelli syndrome associated with haemophagocytic syndrome. Nat. Genet. $25,173-176$

87 Stinchcombe, J.C. et al. (2001) Rab27a is required for regulated secretion in cytotoxic T lymphocytes. J. Cell Biol. 152, 825-833

88 Feldmann, J. et al. (2003) Munc13-4 is essential for cytolytic granules fusion and is mutated in a form of familial hemophagocytic lymphohistiocytosis (FHL3). Cell 115, 461-473

89 zur Stadt, U. et al. (2005) Linkage of familial hemophagocytic lymphohistiocytosis (FHL) type-4 to chromosome 6q24 and identification of mutations in syntaxin 11. Hum. Mol. Genet. 14, 827-834

90 Menager, M.M. et al. (2007) Secretory cytotoxic granule maturation and exocytosis require the effector protein hMunc13-4. Nat. Immunol. $8,257-267$

91 Pickett, J.A. and Edwardson, J.M. (2006) Compound exocytosis; mechanisms and functional significance. Traffic 7, 109-116

92 Robatzek, S. et al. (2006) Ligand-induced endocytosis of the pattern recognition receptor FLS2 in Arabidopsis. Genes Dev. 20, 537-542

93 Gus-Mayer, S. et al. (1998) Local mechanical stimulation induces components of the pathogen defense response in parsley. Proc. Natl. Acad. Sci. U. S. A. 95, 8398-8403

94 Ausubel, F.M. (2005) Are innate immune signaling pathways in plants and animals conserved? Nat. Immunol. 6, 973-979

\section{Reproduction of material from Elsevier articles}

Interested in reproducing part or all of an article published by Elsevier, or one of our article figures? If so, please contact our Global Rights Department with details of how and where the requested material will be used. To submit a permission request online, please visit: 